\title{
Extended Estimations of Neoclassical Transport for the TJ-II Stellarator: The Bootstrap Current
}

\author{
V. Tribaldos* \\ Departamento de Física, Universidad Carlos III de Madrid, 28911 Leganés, Spain \\ C. D. Beidler, Y. Turkin, and H. Maaßberg \\ Max-Planck-Institut für Plasmaphysik, EURATOM Association, D-17491 Greifswald, Germany
}

\begin{abstract}
This work extends previous Monte Carlo estimations of neoclassical transport for the TJ-II stellarator [V. Tribaldos, Phys. Plasmas 81229 (2001)] to include, for the first time, the bootstrap current in low collisionality electron cyclotron resonance heated $(\mathrm{ECRH})$ plasmas. The calculations are based on the mono-energetic coefficients calculated with DKES [W. I. van Rij and S. P. Hirshman, Phys. Fluids B 1, 563 (1989)] and MOCA codes. It is shown that despite the difficulties of calculating these coefficient in the long mean free path for TJ-II configurations the bootstrap current can be accurately estimated thanks to the moderate temperatures measured in this device with and without enforcing momentum conservation [H. Maaßberg and C. D. Beidler and Y. Turkin, Phys. Plasmas 16072504 (2009)]. The computed bootstrap current is found to be in fair agreement with the currents being measured in ECRH regimes. Finally, the effect of the bootstrap current on the rotational transform profile is discussed.
\end{abstract}

\section{INTRODUCTION}

The static (vacuum) confining magnetic field in a stellarator can be obtained solely through a set of coils; i.e. without the need of plasma currents. This allows for steady state operation at the price of a non-symmetrical three-dimensional magnetic field and possibly a complex coil system. The impact of the plasma on its own confinement in these magnetic configurations is very much reduced but it is not completely eliminated. Several mechanisms exist generating currents in the plasma and therefore perturbing the original (designed) magnetic trap. In most stellarators plasma currents are not only not needed but also not wanted. Some of these plasma selfgenerated current effects are known to such a degree that they have been integrated in the stellarator designs since long time ago, like the unavoidable equilibrium currents due to plasma pressure. Minimization of some others current sources can only be enforced partially since they strongly depend on plasma profile details that cannot be self-consistently simulated in advance with enough accuracy like the bootstrap current.

Contrary to other confining schemes, stellarators' flexibility offers the possibility to tailor the rotational transform profile. Two approaches have been used to reduce the effect of undesirable magnetic islands. One consists in squeezing the islands by rapidly crossing the rationals, as is natural in tokamaks. The other depends on finding a gap in the density of rationals, which appears close to low order rational, to place a flat rotational transform profile. This latter solution allows to place large islands at will if enough control is achieved on the shear, like e.g. in island divertor concepts. Unfortunately, this fine control cannot be attained when net currents are present.

\footnotetext{
*Victor.Tribaldos@UC3M.es
}

Apart from unbalanced heating, that in principle could be compensated to a large extent, the main responsible for net plasma currents in stellarators is the neoclassical bootstrap current.

This work will present for the first time a complete estimation of the bootstrap current and the induced modification on the rotational transform in the TJ-II[1] stellarator. In this regard, TJ-II is at an enviable position since: i) as a stellarator, current measurements can be very accurate, ii) its vacuum configurations have low shear and therefore are very sensitive to the currents, iii) is capable of a wide range of rotational transform values $(1.01<t<2.2)$, iv) is equipped with a set of high resolution diagnostics for profile measurements [2,3] and v) currents can be induced with its $\operatorname{ECCD}[4]$ or ohmic transformer systems[5] (each having quite different induced current density profiles). Experimentally, the modest plasma currents $(|I|<2 \mathrm{kA})$ measured in TJ-II for ECRH plasmas have been always attributed to the bootstrap current. The plasma parameter range considered in this work corresponds to ECRH plasmas since, theoretically, bootstrap currents will be negligible for NBI operation because of its much larger collisionality.

The paper is organized as follows: Section II briefly reviews the basic approximations and results of neoclassical theory and shows that a database containing the three mono-energetic coefficients is needed to calculate the bootstrap current. Section III is devoted to the calculation of these coefficients for TJ-II stellarator its difficulties and a discussion on the possibility of describing the bootstrap current accurately. The evaluation of the radial electric field, the bootstrap current and the modification on the rotational transform profile is presented in Section IV with and without enforcing momentum conservation. Finally, Section V summarizes the results and presents conclusions. 


\section{NEOCLASSICAL THEORY}

The starting point to calculate neoclassical effects is the drift kinetic equation (DKE) [6, 7], which is the result of imposing a drift ordering and averaging a full kinetic equation over gyro-scales. The drift ordering, also called small gyro-radius ordering, demands the particle radial drifts, $v_{d}$, to be much smaller than thermal speeds, $v_{t h}$, Larmor radius, $\rho=m v / q B$, much smaller than the characteristic plasma scale lengths, $L$, and particle's bounce frequency, $\omega_{t h}$, much smaller than the cyclotron frequency, $\Omega$, i.e. $v_{d} / v_{t h} \sim \rho / L \sim \omega_{t h} / \Omega=\delta<<1$. Despite the severe simplifications and averages made, the inhomogeneity of the magnetic field on flux surfaces and the non-linearity of the collisional term still make the steady state DKE an intractable non-linear partial differential equation in a five dimensional space. The solution, $f=f(\mathbf{r}, v, p)$, describes the distribution of guiding centers at every point, $\mathbf{r}$, for every speed, $v$, and pitch, $p=\mathbf{v} \cdot \mathbf{B} / v B$, in velocity space. The DKE is further simplified with the local ansatz and the incompressible $\mathbf{E} \times \mathbf{B}$ flow approximations (where $\mathbf{E}=-\nabla \Phi$ is the radial electric field). The local ansatz restricts the equation to a single flux surface, which reduces the dimensionality using the radial position as a parameter rather than a variable. In the drift ordering approximation the kinetic energy is not conserved but its variation is proportional to the divergence of the $\mathbf{E} \times \mathbf{B}$ drift, $V_{E}=\mathbf{E} \times \mathbf{B} / B^{2}$. Whenever $\nabla \cdot V_{E}=0$, as is the case for moderate radial electric fields [8], the total and kinetic energies are conserved, which not only reduces the dimensionality of the DKE again but also allows to use a simplified collision operator only including pitch angle scattering. The final step consists in linearizing the guiding center distribution function with respect to the drift ordering small parameter $\delta<<1$ as $f \approx f_{0}+\delta f_{1}$. The solution to order $\delta^{0}$ is identically satisfied by a local maxwellian $f_{0}=f_{M}=n(r)(m / 2 \pi T(r))^{3 / 2} \exp \left(-m v^{2} / 2 T(r)\right)$ and the resulting DKE equation to order $\delta^{1}$ can be split into two independent equations [9], one for $\hat{f}_{I}$, with a parallel drive

$$
\frac{R_{0}}{v} V\left(\hat{f}_{I}\right)-\frac{R_{0}}{v} \nu L\left(\hat{f}_{I}\right)=-p \frac{B}{B_{0}}
$$

and the other for $\hat{f}_{I I}$, with a radial drive

$$
\frac{R_{0}}{v} V\left(\hat{f}_{I I}\right)-\frac{R_{0}}{v} \nu L\left(\hat{f}_{I I}\right)=-\frac{1}{v_{d}} \frac{d r}{d t}
$$

where $V$ and $L$ are the Vlasov and Lorentz pitch angle collision operators, $\nu$ is the collision frequency, $m$ is the mass, $q$ is the charge, $v$ is the speed, $B_{0}$ is the reference value of the magnetic field on the flux surface (the $B_{00}$ term in the expansion of $B$ in Boozer-coordinates), $R_{0}$ is the major radius, $V_{L}$ is the loop voltage, $v_{d}=m v^{2} /\left(2 q R_{0} B_{0}\right)$ and $d r / d t$ is the first order radial component of the $\nabla B$-drift. Solving these two equations is the central problem of neoclassical theory. A study of eqs. (1) and (2) reveals several important things that are easy to overlook: i) the only radial dependence is contained in the magnetic field, thus these kinetic equations describe a local theory; ii) the movement of particles within the flux surface, contained in the Vlasov operator, is established by the competition of the streaming along magnetic field lines, $p v \mathbf{B} / B$, the $\mathbf{E} \times \mathbf{B}$ drift and the magnetic field mirror term $\propto v \mathbf{B} \cdot \nabla B$, thus for fixed magnetic field it only depends on the normalized drift velocity $v_{E}^{*}=E_{r} / v B_{0}$; iii) the balance between the mirror term and the Lorentz pitch angle collision operator depends on the normalized mean free path $R_{0} \nu / v$; iv) the solution depends on the magnetic field structure but not on its magnitude since it always appears in the combination $\mathbf{B} / B$. Recapitulating, the solution of eqs. (1) and (2) depends on the radial position, through the definition of the normalized magnetic field, and the velocity, which is conserved because of the incompressible approximation, through the two combinations $E_{r} / v B_{0}$ and $R_{0} \nu / v$. From the distribution functions $\hat{f}_{I}$ and $\hat{f}_{I I}$ one can construct all quantities of interest like flux averaged particle or heat fluxes, currents, ..., in the radial or parallel directions by taking different moments and averaging. For example, the flux surface averaged radial particle flux is

$$
\langle\boldsymbol{\Gamma} \cdot \nabla r\rangle=-n\left(L_{11} A_{1}+L_{12} A_{2}+L_{13} A_{3}\right)
$$

or the bootstrap current is

$$
\frac{\langle\mathbf{J} \cdot \mathbf{B}\rangle}{q B_{0}}=-n\left(L_{31} A_{1}+L_{32} A_{2}+L_{33} A_{3}\right)
$$

where the $A_{i}$ terms act as thermodynamic forces since they are related to the radial and parallel derivatives of the maxwellian distribution:

$$
A_{1}=\frac{1}{n} \frac{d n}{d r}-\frac{q E_{r}}{T}-\frac{3}{2} \frac{1}{T} \frac{d T}{d r} ; A_{2}=\frac{1}{T} \frac{d T}{d r} ; A_{3}=\frac{q V_{L}}{R_{0} T}
$$

and where

$$
L_{i j}=\frac{2}{\sqrt{\pi}} \int_{0}^{\infty} d K \sqrt{K} e^{-K} D_{i j} h_{i} h_{j}
$$

with $K=m v^{2} / 2 T, h_{1}=h_{3}=1$ and $h_{2}=K$, and where $D_{i j}$ are the so-called mono-energetic diffusion coefficients

$$
\begin{aligned}
D_{11}=D_{12} & =D_{21}=D_{22}=-\frac{v_{d}^{2} R_{0}}{2 v}\left\langle\int_{-1}^{1} d p \frac{1}{v_{d}} \frac{d r}{d t} \hat{f}_{I I}\right\rangle \\
D_{13} & =D_{23}=-\frac{v_{d} R_{0}}{2}\left\langle\int_{-1}^{1} d p \frac{1}{v_{d}} \frac{d r}{d t} \hat{f}_{I}\right\rangle \\
D_{31} & =D_{32}=-\frac{v_{d} R_{0}}{2}\left\langle\int_{-1}^{1} d p p \frac{B}{B_{0}} \hat{f}_{I I}\right\rangle \\
D_{33} & =-\frac{v R_{0}}{2}\left\langle\int_{-1}^{1} d p p \frac{B}{B_{0}} \hat{f}_{I}\right\rangle
\end{aligned}
$$

that are themselves different moments of the two distribution functions $\hat{f_{I}}$ and $\hat{f_{I I}}$. Now it should be more 
clear the reason why $\hat{f}_{I}\left(\hat{f_{I I}}\right)$ is said to govern the parallel (radial) transport since its antiymmetric (symmetric) part, with respect to the pitch, describes the parallel (radial) fluxes. What it is less clear is that the symmetric (antiymmetric) part of $\hat{f}_{I}\left(\hat{f_{I I}}\right)$ is related to the Ware pinch (bootstrap current) through Onsager symmetry arguments. To compute the lower order neoclassical fluxes only three different coefficients are needed $D_{11}, D_{31}$ and $D_{33}$.

In the neoclassical theory frame, radial transport is the result of averaging over times of the order of the inverse of the collision frequency all possible radial drifts (depending exclusively on $B$ ), or conversely the average radial drift produce by particles moving a distance along the field line of the order of the mean-free path. In some cases the radial average of some, even broad, orbits is very small and disturbing them with collisions results in increased drifts, $D_{11} \propto \nu$ (i.e. like in the collisional Pfirsch-Schlüter (PS) regime or in the banana regime in tokamaks). On the other hand, the characteristic diffusive step size of locally trapped particles is limited by collisions which scatter them out of the local ripple leading to $D_{11} \propto 1 / \nu$ (like various types of trapped particles arising in stellarator fields).

The bootstrap current arises from the distribution function symmetric $\left(\hat{f}_{I}\right)$ or antisymmetric $\left(\hat{f}_{I I}\right)$ on the pitch variable $p$. Thus, in a collision dominated PS regime, where the Vlasov term can be neglected, the symmetric part of $\hat{f}_{I}=0$ (the drive is antisymmetric), or the antisymmetric part of $\hat{f}_{I I}=0$ (the drive is symmetric) the bootstrap current must vanish. On the other limit, deep in the long mean free path, it is possible to show [10] that the bootstrap current is independent of collisionality and it's sign depends on the symmetry of the magnetic field. For toroidal symmetric magnetic fields the bootstrap current flows in the direction of increasing the poloidal magnetic field, thus increasing the rotational transform, while for helically symmetric fields the bootstrap current reduces the poloidal magnetic field and the rotational transform. See for example the NCSX (toroidally quasi-symmetric) and HSX (helical quasi-symmetric) in ref.[9]. The situation at intermediate collisionalities for purely symmetric fields is a transition from the null value at high collisionality to the plateau value in the collisionless limit. For non-symmetric devices this transition is far more troublesome. What it is usually seen is a bootstrap coefficient dominated by short range helically trapped particles at higher collisionalities, flowing in the direction of decreasing the poloidal magnetic field, followed by a transition at smaller collisionalities (larger mean-free paths) where the toroidal terms dominate the drifts producing an increase in the poloidal magnetic field. This different behavior has been used in the design of Wendelstein-7X stellarator to reduce the total bootstrap current [11].

The third mono-energetic coefficient, $D_{33}$, depends solely on the antiymmetric part of $\hat{f}_{I}$, solution of Eq. 1 . $D_{33}$ measures the response of particles to a parallel elec- tric field, which is a competition between the Vlasov and collisional terms in Eq. 1. The number of particles flowing along field lines, the $p v \mathbf{B} / B$ term in the Vlasov operator, is reduced because of the trapping caused by the mirror term, $\mathbf{B} \cdot \nabla B$, and the collisions that stochastically change their pitch angle. Collisions always produce a reduction of $D_{33}$, which is a monotonically decreasing function of collisionality, but the mirror term only has an effect on ripple structures smaller than the mean free path. The impact of trapped particles is negligible at high collisionalities and saturates in the lmfp.

\section{THE THREE MONO-ENERGETIC DIFFUSION COEFFICIENTS}

A quick inspection of Eq. (4) would suggest that to estimate the neoclassical parallel flow (i.e. the bootstrap current $)$ in a stellarator $\left(A_{3}\right.$ should be zero since in principle no loop voltage, $V_{L}$ is necessary) it would be sufficient to solve either Eq. (1) to obtain $\hat{f}_{I}$ or Eq. (2) for getting $\hat{f_{I I}}$ since only the $D_{13}$ or $D_{31}$ mono-energetic coefficients are necessary. Unfortunately, these coefficients depend strongly on the value of the drift speed $V_{E}=E_{r} / v B$, i.e. the radial electric field. This dependence is so pronounced that an independent method is needed to determine the radial electric field. As is customary in nonsymmetric magnetic field devices, the neoclassical radial electric field is obtained by imposing ambipolarity, i.e. the same electron and ion flux surface average particle fluxes, or more generally that $\Gamma_{e}=\sum_{\alpha} q_{\alpha} \Gamma_{\alpha}$ (for all ion species $\alpha$ ). Remembering Eq. (3) this means that the $D_{11}$ coefficient is also needed. Actually, in Section IV it is shown that to enforce momentum conservation, which was not preserved with the Lorentz collision operator, the $D_{33}$ coefficient is also needed.

Calculating the bootstrap current for a given magnetic configuration requires creating a database of the three mono-energetic diffusion coefficients, thus solving both eqs.(1) and (2), for several radial positions, normalized drift velocities, $E_{r} / v B_{0}$ and mean-free-paths, $R_{0} \nu / v$. Several numerical techniques are nowadays available for the calculation of the mono-energetic diffusion coefficients [9] each with its strengths and drawbacks: i) analytical calculations [6, 7]; ii) spectral decomposition $[12,13]$ methods; iii) field line integration $[14,15]$ techniques, and iv) Monte Carlo [16-21] simulations. However, not all of them are suited for the TJ-II stellarator because of its complex magnetic field structure; because they don't include the effect of the radial electric field; or because they can only compute one of the coefficients effectively. To create the database needed for the purpose of this work the DKES code was used [12, 13] since it gives the three mono-energetic coefficients, along with their uncertainties, for general magnetic fields. It was found that the convergence and precision of DKES code estimates of $D_{11}$ and $D_{31}$ deteriorates with increasing mean free path and depending on the number of 
modes necessary to accurately described the magnetic field a point is reached where even a small reduction in the calculation uncertainty becomes computationally prohibitive.

The three mono-energetic coefficients, solutions of the code, share limitations with their originating kinetic equations, namely: transport is considered local and diffusive, the $\mathbf{V}_{E}=\mathbf{E} \times \mathbf{B} / B^{2}$ drift is considered incompressible, $\nabla \cdot \mathbf{V}_{E}=0$ and collisions are approximated by the Lorentz operator. Non-local effects lie outside the scope of this paper, although they have been described for the TJ-II stellarator [22]. The assumption of incompressibility of the $\mathbf{E} \times \mathbf{B}$ drift limits [8] the validity of the results to radial electric fields smaller than the first electric field resonance $E_{r e s}=t v B r / R$, where $t$ is the rotational transform, $r$ the minor radius of the flux surface and $R$ the major radius of the device. Finally, the Lorentz operator approximation requires a treatment, using the generated DKES database, to restore momentum conservation that will be discussed in the next section.

To create a database with the three mono-energetic coefficients the first step is to obtain the magnetic field spectrum of a given equilibrium configuration in Boozer magnetic co-ordinates. The VMEC [23] and JMC [24] codes were used to obtain the equilibrium configuration and making the VMEC to Boozer co-ordinate transformation. A database was created comprising around 2850 DKES calculations divided into seven radial positions, $r / a$, around fifteen inverse mean-free paths, $\nu / v$, and many values of the parameter $E_{r} / v B$. The radial electric field grid was made sufficiently fine to resolve, at least, the first electric field resonance $E_{\text {res }}$. The three normalized diffusion coefficients are plotted versus the normalized collisionality, $\nu^{*} \equiv R_{0} \nu /(t v)$, at half radius in Fig. (1) for several values of the radial electric field. This is the first time that the bootstrap, $D_{31}$, and conductivity, $D_{33}$, mono-energetic coefficients are published for TJII stellarator. The lack of DKES values in the $\operatorname{lmfp}$ was partly mitigated using $D_{11}$ results from the Monte Carlo code MOCA and for $D_{33}$ using the extrapolation to the fraction of passing particles, $f_{p}=\frac{3}{4}\left\langle h^{2}\right\rangle \int_{0}^{1} \frac{\lambda d \lambda}{\left\langle(1-\lambda h)^{1 / 2}\right\rangle}$ with, $h=B / B_{\max }$. The values shown are normalized as in [9]: $D_{11}^{*}=D_{11} / D_{11}^{p}$ with $D_{11}^{p}=\pi v_{d}^{2} R_{0} / 4 v t ; D_{31}^{*}=$ $D_{31} / D_{31}^{b}$ with $D_{31}^{b}=2 v_{d} R_{0} f_{p} / 3 v t ; D_{33}^{*}=D_{33} / D_{33}^{P S}$, with $D_{33}^{P S}=v^{2}\left\langle B^{2}\right\rangle / 3 \nu B_{0}^{2}$. Notice the logarithmic scale in $D_{11}^{*}$, the linear scale in the bootstrap coefficient, $D_{31}^{*}$ and how error bars get larger at smaller collisionalities and that the $D_{33}^{*}$ is independent on the radial electric field. In performing the energy convolutions with the Maxwellian distribution function mono-energetic coefficients are required not only at intermediate grid points (interpolation) but also outside of the database (extrapolation). The interpolation part was carried out with a MLP1 type neural network (NN) as in Ref. [17] but now including the other two mono-energetic coefficients, $D_{31}$ and $D_{33}$. The NN performs a very quick and smooth interpolation of the three coefficients on

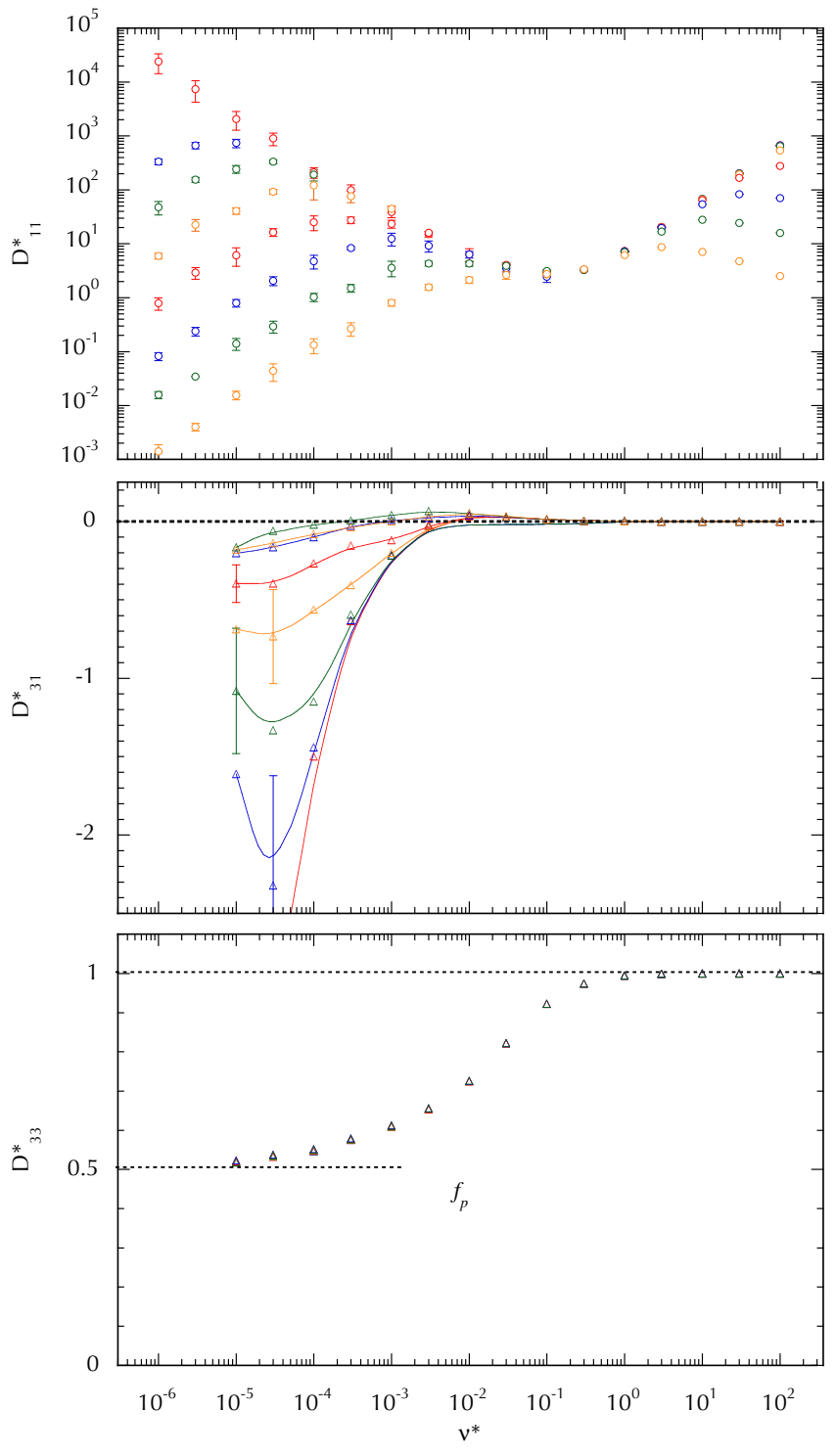

FIG. 1. (Color online) Normalized mono-energetic coefficients versus collisionality at half radius for the standard configuration of TJ-II stellarator and $E_{r} / v B=0,1 \times 10^{-5}, 3 \times 10^{-5}$, $1 \times 10^{-4}, 3 \times 10^{-4}, 1 \times 10^{-3}, 3 \times 10^{-3}$, and $1 \times 10^{-2}$. MOCA data is shown for $D_{11}^{*}$ and DKES results for $D_{31}^{*}$ and $D_{33}^{*}$.

the radial, $r / a$, electric field, $E_{r} / v B$ and collisionality, $\nu^{*}$, dependences. On the other hand, the extrapolation problem was treated case by case. The radial direction poses only a problem towards the axis for $D_{11}$ in the lmfp. In this limit a least-squared fit to the numerical results with the known analytical collisional and electric field dependences, $D_{11}=D_{1 / \nu} \propto 1 / \nu^{*}, D_{11}=D_{\sqrt{\nu}} \propto$ $\sqrt{\nu^{*}}\left(v B_{0} / E_{r}\right)^{3 / 2}$ and $D_{11}=D_{\nu} \propto \nu^{*}\left(v B_{0} / E_{r}\right)^{2}$, is used in the extrapolation to the axis. For the electric field, the limit $E_{r} \rightarrow 0$ only affects $D_{11}$ and $D_{31}$ in the $l m f p$ ( $D_{33}$ is independent on the radial electric field) and it will be treated below in the collisionless limit discussion. 
In the large radial electric field limit the mono-energetic coefficients are set to their maximum calculated values, i.e. around the first electric field resonance to avoid difficulties with the incompressible $E \times B$ approximation [8]. For the collisionality extrapolations, the Pfirsch-Schlüter regime is usually not a problem except for $D_{11}$ at high values of $E_{r} / v B$, like those found for impurities, which are not considered in this work. The collisionless limit problem for $D_{11}$ is solved with the use of the Monte Carlo solution of MOCA [17]. Obtaining the behavior of $D_{31}$ is not possible since no general, accurate and fast solution is known; a problem that is exacerbated for the very broad field spectra of TJ-II configurations. The lack of accurate solutions at very low collisionalities is not a serious problem for the calculations because of the plasma parameters found in TJ-II [25]. In estimating the thermal coefficients Eq. 5 one has to evaluate the energy convolution, which is the product of three terms: i) the monoenergetic coefficient, $D_{i j}\left(r / a, \nu / v, E_{r} / v B\right)$, a power of the kinetic energy, $K=\left(v / v_{t h}\right)^{2},\left(v_{t h}=\sqrt{2 T / m}\right)$ and an exponentially decreasing function of the kinetic energy, $\exp \left[-\left(v / v_{t h}\right)^{2}\right]$, i.e. the maxwellian distribution function. The integral in kinetic energy, or speed, from zero to infinity (relativistic effect are negligible) translates into the mono-energetic coefficients' variation in inverse mean-free-path, $\nu / v$, from collisionless (infinite mean free path) to fully collisional (zero mean free path). As shown in the literature $[6,9]$ and also in Fig. 1 the dependence of the three coefficients on the mean free path is quite different. In the collisional limit $D_{11}$ scales with $\nu^{*}, D_{31}$ tends to zero as $1 / \nu^{* 2}$ and $D_{33}$ scales with $1 / \nu^{*}$ (what is plotted in Fig. 1 is $D_{33}^{*}$ not $\left.D_{33}\right)$. Therefore, the argument of the integrals involving all coefficients tend to zero because of the $\left(v / v_{t h}\right)^{3}$ factor. In the collisionless limit, both $D_{11}$ and $D_{33}$ scale with $1 / \nu^{*}$ for $E_{r}=0$ and $D_{31}$ saturates at a plateau value [26]. Here the maxwellian exponential decrease in the number of particles with energy is much faster than the diffusion coefficients increase with the mean free path. However, the unfavorable $1 / \nu^{*}$ scaling of $D_{11}$ and $D_{33}$ extends the region contributing to the integral to smaller values in collisionality and calls for precise calculations of these coefficients deeper in the lmfp. On the other hand, the contribution of $D_{31}$ in the lmfp has a smaller impact on the result of the thermal convolution. How deep and how accurate the calculation has to be depends on the maxwellian term, i.e. the local temperature. In this regards the low temperatures found in TJ-II plasma operation balance the troubles associated with its broad magnetic field spectrum and the associated difficulties for accurately calculate $D_{31}$.

To clarify this effect the argument of the integral defining $L_{32}$ is plotted in Fig. 2 for electrons and ions versus the normalized collisionality at $r / a=0.2,0.5$ and 0.8 and the conditions of Case 1 of next section. The collisional dependence starts and ends at zero because of the $v / v_{t h}$ power and the maxwellian terms. In between, it is controlled by $D_{31}$, which is zero in the PS regime, has the sign of decreasing the absolute value of the rotational transform at intermediate collisionalities because of the non-axisymmetric field terms, and in the $\operatorname{lmf} p$ has the sign of increasing $|t|$ due to axi-symmetric contributions. The unusual signs of the coefficients in Fig. 2 e.g. the electron (ion) contribution is positive (negative) in the lmfp is not in contradiction with the above discussion. The signs are reversed for negatively charged particles, see the normalization of $D_{31}^{*}$. In TJ-II, bootstrap current flows in the direction of increasing the poloidal component of the magnetic field at low collisionalities. The reason for the bizarre sign is that the rotational transform in TJ-II is negative, see Fig.1 in [17], a positive toroidal current decreases the poloidal magnetic field and thus $t$.

A rapid comparison of the result for $r / a=0.5$, with the middle plot in Fig. 1 shows that for electrons the maximum contribution to the integral comes from the region around $\nu^{*} \approx 10^{-3}-10^{-4}$, where the uncertainty in the calculation of $D_{31}$ coefficient is moderate for the ambipolar electric field considered. Notice that this is the worst possible case since $L_{32}$ decreases more slowly with decreasing collisionality than $L_{31}$ and $L_{33}$ because of the $h_{2}=\left(v / v_{t h}\right)^{2}$ term. The radial dependences are explained considering the electron and ion temperature profiles and the larger non-axisymmetric field contribution in TJ-II closer to the axis, that increases the positive part of $D_{31}^{*}$ and extends it to smaller collisionalities. This effect is noticeable in Fig. 2 at $r / a=0.2$ where the higher temperature of electrons shifts the contributing region to lower collisionalities and the larger central helically symmetric contribution amplifies the negative part. The odd behavior seen for the ions at $r / a=0.5$ is due to its higher temperature.

Let's finish this section with the final expression that will be used for calculating the local bootstrap current density for particles of type $\alpha$ from Eq. (4), it is:

$$
j_{b}^{\alpha}(r)=-q_{\alpha} n_{\alpha}(r)\left[L_{31}^{\alpha}(r) A_{1}^{\alpha}(r)+L_{32}^{\alpha}(r) A_{2}^{\alpha}(r)\right] .
$$

Notice that the $L_{i j}$ coefficients are functions of position through the local density and temperature dependence on collisionality and radial electric field.

\section{RESULTS}

There are two heating scenarios in TJ-II operation: i) Electron Cyclotron Resonance Heating (ECRH) and ii) Neutral Beam Injection (NBI) heating. ECRH is used for plasma startup and heating below the cut-off density, which is $n_{\text {cut-off }} \approx 1.7 \times 10^{19} \mathrm{~m}^{-3}$ for the $f=53.2 \mathrm{GHz}$ gyrotron setup. NBI is used with a sufficiently dense and hot plasma target and allows overcoming the cut-off density. In either case, the heating power is coupled to the electrons (in NBI because of the high beam energy $E_{\text {beam }}=35 \mathrm{keV}$ ) The ion temperature is always limited to about $T_{i} \approx 200 \mathrm{eV}$; at low densities because of the bad electron-ion coupling [31] and at higher densities because 

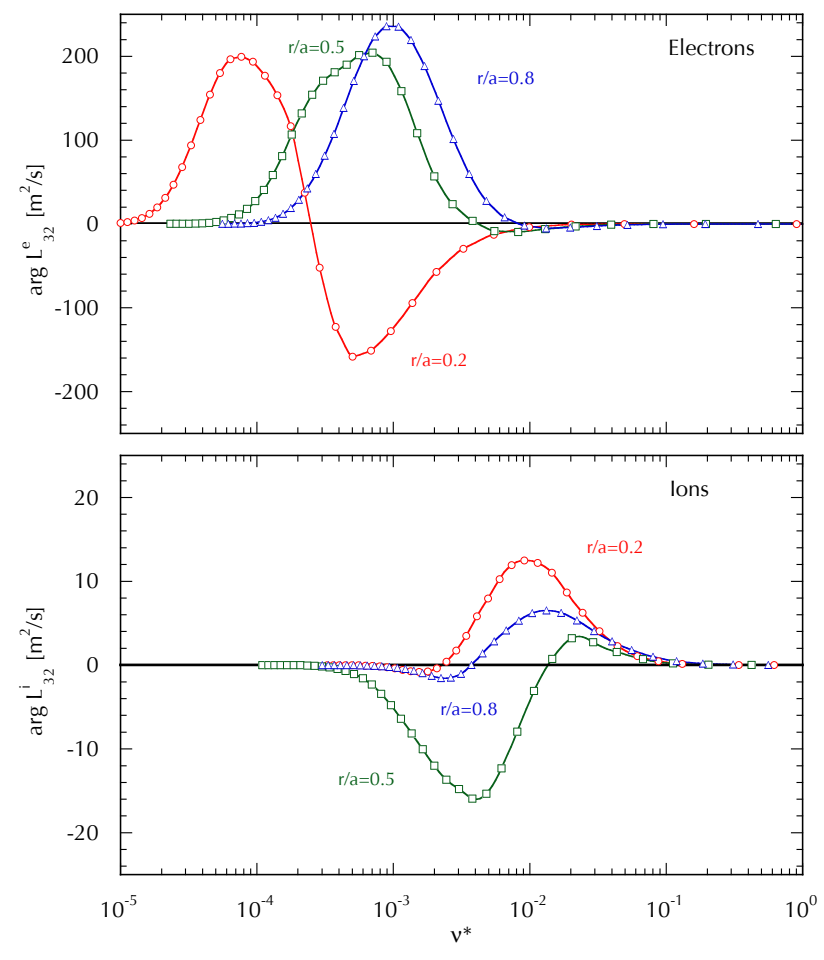

FIG. 2. (Color online) Argument of the integral defining $L_{32}$, see Eq. 5, versus normalized collisionality for electrons (top) and ions (bottom) at $r / a=0.2$ (circles), 0.5 (squares) and 0.8 (triangles) and their local ambipolar radial electric field corresponding to the plasma parameters of the first profile considered in next section. Points are not equally spaced because of the Gauss-Laguerre integration technique used

of the large ion transport [17]. For central ECRH deposition, the electron temperature increases, from around $T_{e} \approx 200 \mathrm{eV}$ in NBI up to $T_{e} \approx 1.5 \mathrm{keV}$ in ECRH and gets more peaked with decreasing density [32]. For these plasma parameters one expects bootstrap currents to be mainly driven by electrons and being only sizable in low density ECRH operation. TJ-II was originally designed with enough flexibility to produce plasma configurations with different rotational transforms and, to a minor extent, different shears. These plasma parameter change from configuration to configuration is accompanied with plasmas at different positions inside the vacuum vessel and with different shapes and sizes [4, 28]. For this first calculation of the bootstrap current and given work involved in obtaining the mono-energetic coefficients and the uncertainties in the plasma profiles it was decided to calculate the database just for the standard configuration of TJ-II and wait for experimental evidences to justify further theoretical studies. Results of Ref. [29], where around 4000 configurations were analyzed seeking for a transport optimized configuration, suggest that, at least, $D_{11}$ is a smooth function of the configuration although strong variations cannot be ruled out especially for very small rounded plasmas and close to rational sur-
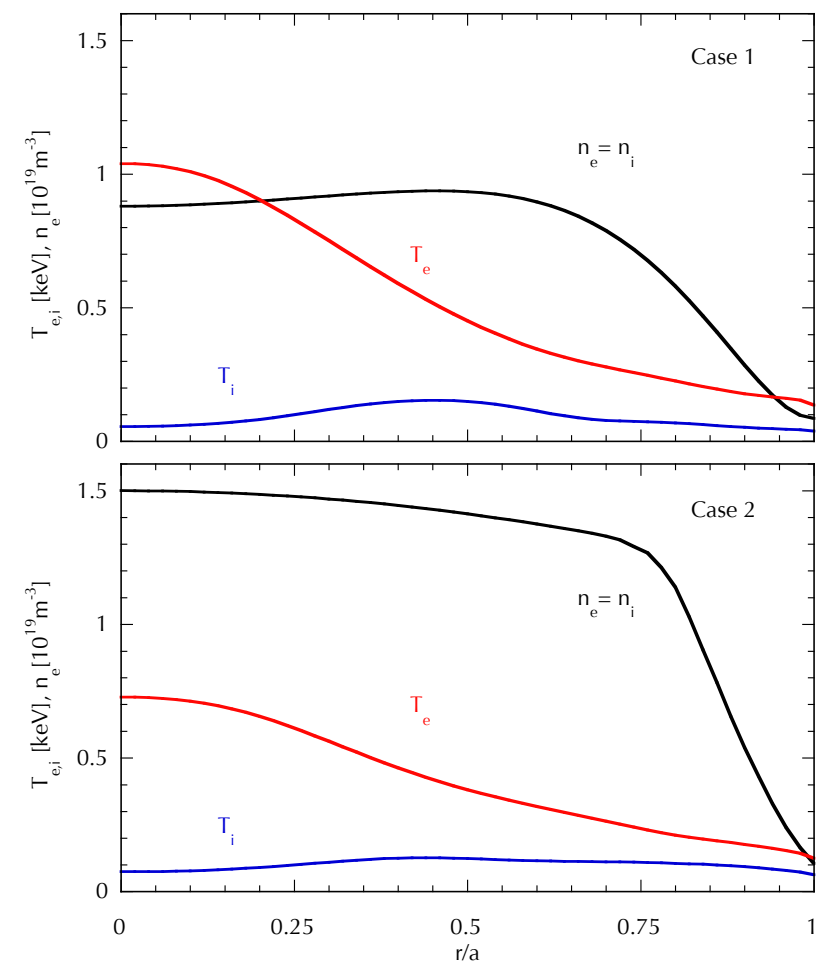

FIG. 3. (Color online) Density and electron and ion temperature profiles for Case 1 (top) and Case 2 (bottom).

faces. As for the $\beta$ dependence, there is an unfavorable effective helical ripple dependence with increasing $\beta$ despite the very mild modification of the flux surfaces with plasmas pressure [30].

Two plasma scenarios were selected as examples for calculating all neoclassical estimates like the ambipolar radial electric field, the electron and ion particle and energy fluxes and bootstrap current densities. Both scenarios were simulated with the predictive transport code of Ref. [33]. In the predictive simulations density and ECRH power deposition profiles (with $P_{E C R H}=200$ $\mathrm{kW}$ ) were fixed and the electron and ion temperatures were obtained solving the energy balance equations taking into account the neoclassical contributions, for the ambipolar radial electric field, plus an edge anomalous contribution. Ion and electron density profiles were fixed, and set equal to each other $(Z=1)$ because of the uncertainties in the recycling, the very small neoclassical contributions at the edge and the difficulties in modeling the anomalous particle channel. The first set of profiles, Case 1, is the same as the one presented in Ref. [34], the second one, called Case 2, corresponds to a higher density ECRH operation. The density and temperature profiles are plotted in Fig. 3. These two cases correspond approximately to the ECRH operation regimes described in Refs. $[35,36]$ and where the net current was measured. Unfortunately, it was not possible to use these experimental results directly since not all necessary pro- 


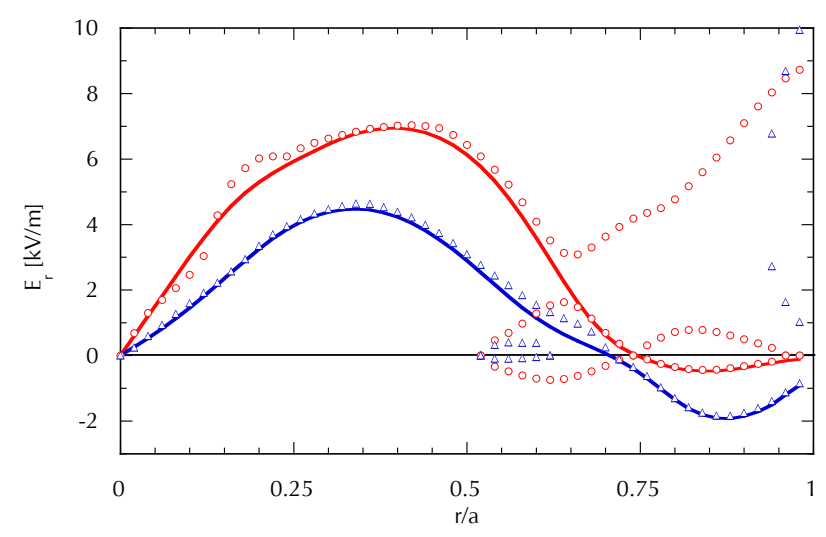

FIG. 4. (Color online) Radial electric field profiles for the Case 1 (circles) and Case 2 (triangles).

files were available (ion temperature and $Z_{\text {eff }}$ profiles) or were not available with the required accuracy for all radii (edge electron density and temperature).

Since the impact of the Lorentz collision operator on the perpendicular particle and energy transport (and the radial electric field) is of minor importance for nonaxisymmetric devices except in the PS regime [27], the procedure to make neoclassical estimates for these two scenarios is the same as the one presented in Ref. [17]: 1) solve the ambipolar condition $\sum_{\alpha} Z_{\alpha} \Gamma_{\alpha}=0$ for the particle fluxes

$$
\Gamma_{\alpha}\left(r, E_{r}\right)=-n_{\alpha}(r)\left[L_{11}^{\alpha}(r) A_{1}^{\alpha}(r)+L_{12}^{\alpha}(r) A_{2}^{\alpha}\right]
$$

where the Ware pinch is negligible, and use the solution to obtain the ambipolar particle flux and the electron and ion heat fluxes. In general, when there are all type of thermodynamic forces, as in the outer part of the plasma, not even the sign of the radial electric field can be anticipated and the non-linear equation has to be solved numerically (e.g. Fig. 8 of Ref. [17]), possibly leading to multiple solutions. In Fig. 4 the solutions to the ambipolar condition are plotted for both scenarios ( the result for Case 1 is equal to Fig. 4 of Ref. [34]). Only one solution with positive $E_{r}$ (electron root) is found in the plasma core followed by three solutions (there is always an odd number of solutions) at outer radius. The predicted radial electric field is obtained from the solution of a diffusion equation for the radial electric field, see Ref. [34] for details. It is worth stressing the fair agreement between the results of Fig. 4 and the radial electric field measured [37] by the HIBP diagnostic in TJ-II for similar plasma parameters.

Once the radial electric field was obtained it is possible to make all type of neoclassical estimates. The ambipolar particle flux, $\Gamma$, is plotted along with the electron, $Q_{e}$, and ion, $Q_{i}$, heat fluxes in Fig. 5. Where the radial
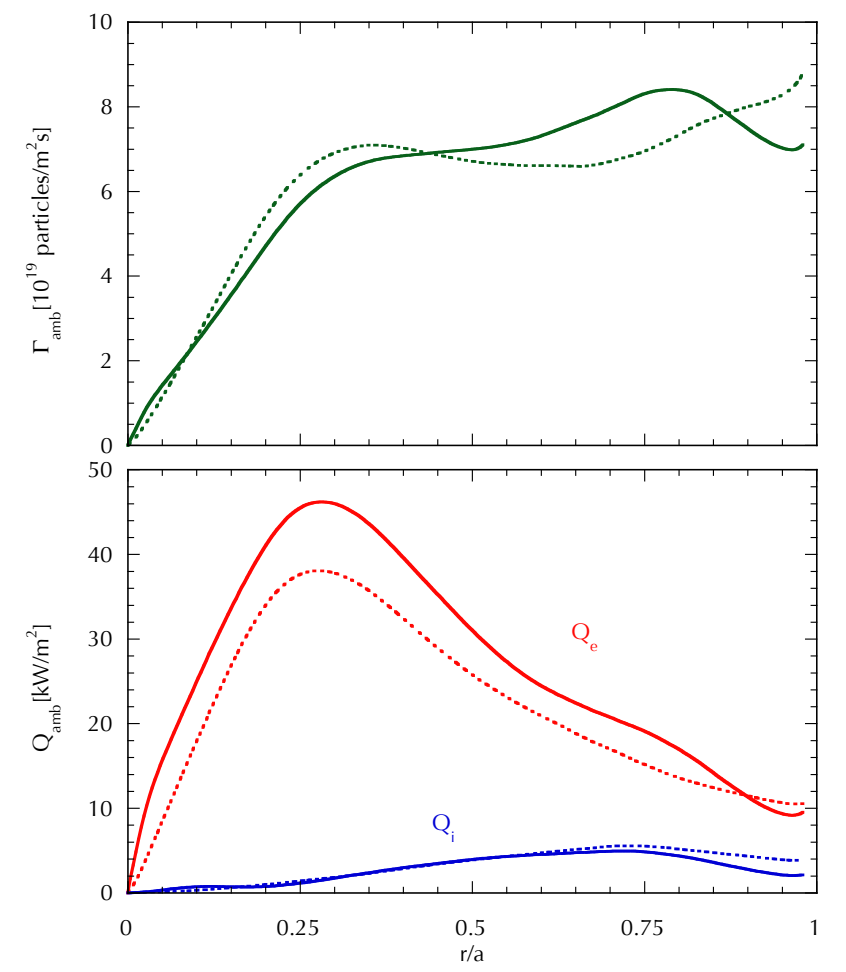

FIG. 5. (Color online) Ambipolar particle (top) and electron and ion heat (bottom) flux profiles for Case 1 (solid) and Case 2 (dotted).

energy fluxes are defined as:

$$
Q_{\alpha}(r)=-n_{\alpha}(r) T_{\alpha}(r)\left[L_{21}^{\alpha}(r) A_{1}^{\alpha}(r)+L_{22}^{\alpha}(r) A_{2}^{\alpha}(r)\right]
$$

where, again, the term related to the loop voltage was omitted. Integrating these profiles one can calculate derived quantities like the local particle and energy confinement times, which in the cylindrical approximation leads for the ambipolar particle confinement time:

$$
\tau_{p}(r)=\frac{4 \pi^{2} R \int_{0}^{r} n\left(r^{\prime}\right) r^{\prime} d r^{\prime}}{4 \pi^{2} r R \Gamma(r)}
$$

and the energy confinement time

$$
\tau_{E}(r)=\frac{4 \pi^{2} R \int_{0}^{r} \frac{3}{2} n\left(r^{\prime}\right)\left[T_{e}\left(r^{\prime}\right)+T_{i}\left(r^{\prime}\right)\right] r^{\prime} d r^{\prime}}{4 \pi^{2} r R\left[Q_{e}(r)+Q_{i}(r)\right]},
$$

where $\Gamma$ and $Q_{\alpha}$ are given by Eq. (8) and (9). A comparison of these confinement times with experimental analysis reveals a difficulty: experimentally, particle and heat fluxes increase towards the edge whereas neoclassical fluxes decrease towards the edge (are proportional to the local density and temperature and strongly decreasing functions of collisionality). So far, there is no satisfactory explanation for this anomalous discrepancy in stellarators. To partly avoid this difficulty, here (see Table I) the particle and energy confinement times are compared at 
$2 / 3$ of the plasma radius for the both scenarios. The values obtained are very close to those obtained experimentally in Ref. [38] in which the particle confinement time was found to be approximately $\tau_{P} \approx 10^{5} \times\left(P_{i n} / \bar{n}_{e}\right)^{-1.7}$ $\mathrm{ms}$, where $P_{\text {in }}$ is the ECRH injected power in $\mathrm{kW}$ and $\bar{n}_{e}$ the line integrated density in $10^{19} \mathrm{~m}^{-3}$. They are also in agreement with a predictive transport code [39] using the plasma profiles, absolutely calibrated $H_{\alpha}$ signals and EIRENE code simulations to estimate flux surface averaged particle sources [40]. The fair agreement found between the neoclassical energy confinement time and the International Stellarator Scaling ISS04 [41] points to the importance of the neoclassical channel for the TJ-II.

\begin{tabular}{|r|r|r|r|r|}
\hline & \multicolumn{2}{|c|}{ Case 1 } & \multicolumn{2}{|c|}{ Case 2 } \\
\hline$\tau_{P}[m s]$ & Neo. & Ref. [38] & Neo. & Ref. [38] \\
& 6.5 & 7.7 & 12.0 & 17.6 \\
\hline$\tau_{E}[m s]$ & Neo. & ISS04 & Neo. & ISS04 \\
& 3.0 & 2.2 & 4.5 & 2.8 \\
\hline momentum & without & with & without & with \\
$I_{e}[\mathrm{kA}]$ & -2.36 & -2.39 & -2.00 & -2.01 \\
$I_{i}[\mathrm{kA}]$ & 0.07 & 0.62 & 0.16 & 0.67 \\
$I_{\text {tot }}[\mathrm{kA}]$ & -2.29 & -1.77 & -1.84 & -1.35 \\
\hline
\end{tabular}

TABLE I. Particle and energy confinement times and Bootstrap current for the two scenarios considered. $\tau_{E}^{\text {ISS04 }}=0.134 f_{\text {TJ-II }} a^{2.28} R^{0.64} P^{-0.61} \bar{n}^{0.54} B^{0.84} t_{2 / 3}^{0.41}$ and for TJ-II: $f_{\text {TJ-II }}=0.25, a=0.18 \mathrm{~m}, R=1.5 \mathrm{~m}, P=0.2$ MW, $B=0.95 \mathrm{~T}, t_{2 / 3}=1.48, \bar{n}_{\text {Case } 1}=0.76 \times 10^{19} \mathrm{~m}^{-3}$, $\bar{n}_{\text {Case } 2}=1.24 \times 10^{19} \mathrm{~m}^{-3}$

The procedure implemented in this work to correct the bootstrap estimation given by Eq. 7 is described in Sec. $\mathrm{V}$ of in Ref. [27] which is based on a moment equation expansion of the DKE. The electron and ion corrections are coupled to each other and are obtained solving a system of linear equations of size equal to the number of species times the number of Sonine polynomials (which is three for the simulations presented here). The electron and ion bootstrap current density profiles are presented in Fig. 6 with and without imposing momentum conservation for the high and low density ECRH scenarios. The electron bootstrap current density is almost everywhere negative and larger, in absolute value, than the ion contribution. This can be anticipated from the $D_{31}$ dependence on collisionality, see Fig. 1, and the disparate electron and ion collisionalities. In Case 2 the electron bootstrap current density is positive in the plasma core because of a combination of low temperature and small gradients. The momentum correction on the individual electron and ion channels is a tradeoff between their parallel flows and their collisional coupling. For the considered parameters, the impact of momentum correction on the ion contribution is rather large; the parallel viscous damping of the ion flow at higher collisionalities is described by the effective trapped particle fraction, $1-D_{33}^{*}$ (see Fig.1). On the other hand, the electron flow is collisionally coupled to the ion flow and partly compensates the strong effect of the ion momentum correction (most pronounced in Fig. 6 at inner radius for Case 1). These effects, i.e. the huge ion correction and the electron partial cancellation, can be seen in Fig. 6 where the ion and electron current densities are shown with and without the momentum corrections. The overall effect of momentum conservation is a reduction in the total bootstrap current. In Ref. [27] it is explained the different effect of the momentum conservation on the bootstrap current depending on the importance of the density or temperature gradients. For temperature gradient dominated profiles a significant reduction is found when considering a collision operator conserving momentum, whereas density gradients produce an increase in the bootstrap current. The reduction found here for both cases is in agreement with these results since both are mainly electron temperature gradient dominated. The results obtained are consistent with the neoclassical ordering since the radial electric field is small enough to neglect radial momentum transport effects (even for the lowest collisionality and the central electron root $E_{r} R / t v_{t h}^{i} r B$ is below 0.2) and the ion parallel flow is small compared with the ion thermal velocity, $j_{i} / q_{i} n_{i} v_{t h}^{i}<0.3$. It is difficult to compare the bootstrap current values obtained with the net currents measured during ECRH operation, ranging from -0.5 to -1 . $\mathrm{kA}^{35,36}$ since on the one hand experimental values might be influenced by ECCD and on the other hand because of the sensitivity of the total current on the edge profiles and the $Z=1$ value. Nevertheless, neoclassical estimations have the same current sign and are less than a factor of two larger than the experimental measurements. More remarkable is the fact that better agreement is found when momentum corrections are included.

The impact of the bootstrap current on the rotational transform can be calculated in several ways: with a cylindrical approximation, through the geometrical factors of Ref. [42] or by directly including the currents in a 3D MHD code. In the past [4] no significant difference was found for TJ-II between the full MHD and the simple cylindrical approach, so this latter was used here, $t=t_{0}+\Delta t$, where $t_{0}$ is the vacuum rotational transform and the modification, $\Delta t$, due to the plasma current density $j$ is:

$$
\Delta t(r) \approx \frac{\mu_{0} R}{B r^{2}} \int_{0}^{r} j\left(r^{\prime}\right) r^{\prime} d r^{\prime}
$$

Using the bootstrap current density profiles obtained (see Fig. 6), which are small or a bit positive close to the axis and negative at outer radius on can anticipate that $\Delta t=0$ on axis (there are no gradients on axis); will reduce the absolute value of the rotational transform close to the axis, $\Delta t>0$, and will increase $t$ towards the edge, $\Delta t<0$. In Fig. 7 the original and the modified $t$ profiles are plotted with and without the momentum correction for both cases analyzed. These results are in accordance with the experimental plasma currents measured at TJII during ECRH operation, without electron cyclotron current drive (ECCD), that are always negative [25]. For 

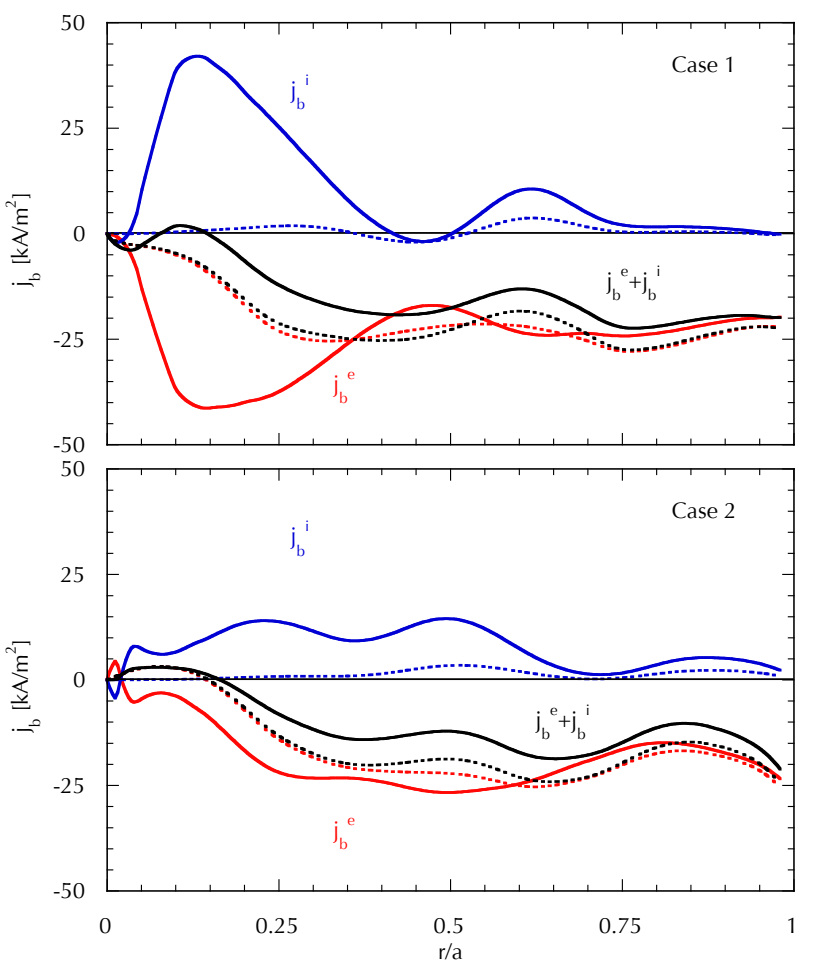

FIG. 6. (Color online) Bootstrap current density profile with (solid) and without (dotted) imposing momentum conservation for Case 1 (top) and Case 2 (bottom)

these conditions the bootstrap current reduces the absolute value of the rotational transform close to the axis and increases it towards the edge. As a consequence, the rotational transform radial variation, i.e. the shear, $t^{\prime}=d t / d r$, increases with respect to the vacuum configuration. Finally, it is worth stressing that once the database with the three mono-energetic transport coefficient is computed the calculation of all the neoclassical estimations of this section for a given set of plasma profiles takes half a second on a laptop computer .

\section{CONCLUSIONS}

For the first time bootstrap current has been computed for TJ-II stellarator with the DKES and MOCA codes despite the numerical difficulties found in the calculation of the mono-energetic transport coefficients in the long mean free path for this device. This was made possible because of the smooth dependence of the bootstrap coefficient on the collisionality and radial electric field and the moderate electron and ion temperatures found in the routine ECRH operation in TJ-II. Neoclassical estimates of: ambipolar radial electric field, ambipolar particle flux, electron and ion heat fluxes, particle and energy confinement times are presented for two ECRH scenarios obtained with a predictive transport code. A

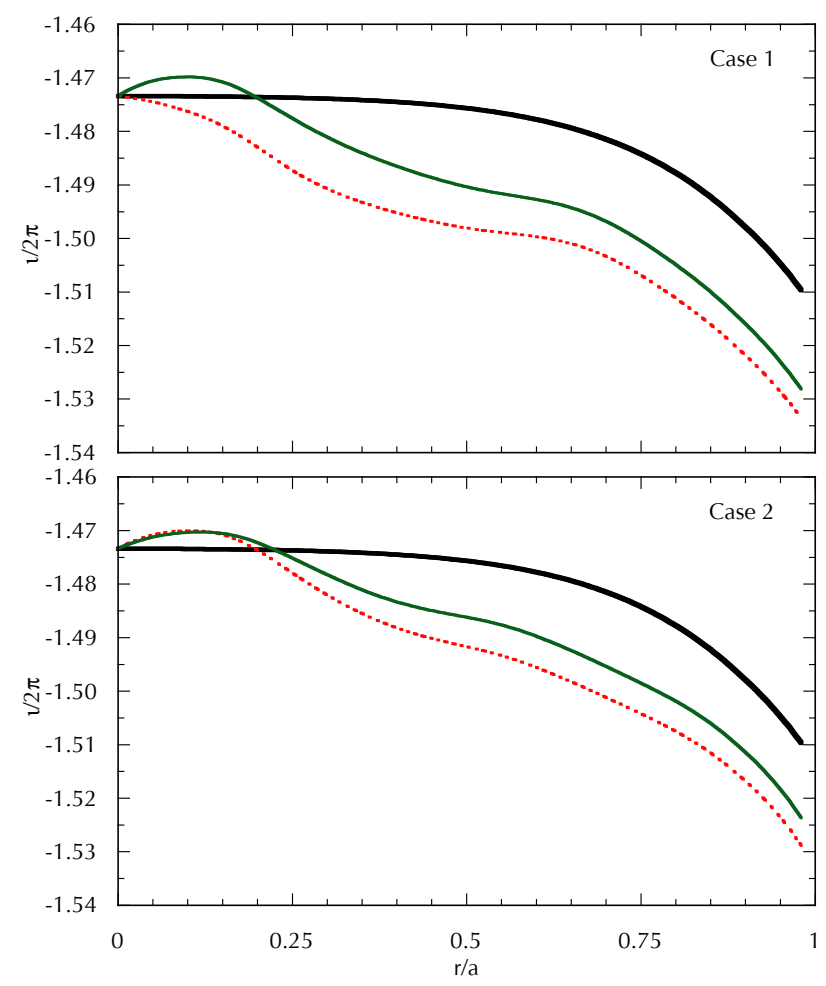

FIG. 7. (Color online) Rotational transform profiles in vacuum (thick solid) and including the modification due to bootstrap current with (solid) and without (dotted) imposing momentum conservation for Case 1 (top) and Case 2 (bottom).

remarkable agreement is found between neoclassical particle confinement times and those calculated from a combination of experimental profiles and neutral code simulations and between the energy confinement times and the ISS04 scaling law estimation. The bootstrap current density is calculated with and without applying a momentum correction technique based on moment equations. The results exhibit modest current densities in the central plasma region becoming more negative at outer radius due to the, low collisional, electron contribution and a total negative integrated bootstrap current. The effect of the momentum correction technique is a slight reduction of the integrated current. The obtained bootstrap currents, $I_{b} \approx-1.5 \mathrm{kA}$ are close to the plasma currents usually measured in TJ-II during ECRH operation. The modification of the rotational transform has been calculated with a cylindrical approximation and shows a reduction, in absolute value, close to the axis and a later increase, also in absolute value, at outer radius. Rational values of the rotational transform that were thought to lie outside the plasma can be found inside the configuration, both in the plasma core or at the edge. The modified plasma configurations have a much larger rotational transform shear, $t^{\prime}=d t / d r$, up to $3 a / 4$, possibly with a multivalued $t$ close to the axis, and almost the same shear at outer positions. 
These conclusions are largely independent of the profile details, as many other simulations that have not been included here indicate. The neoclassical analysis presented in this work allows a fast and systematic comparison with experimental radial electric field profiles and plasma currents and should be taken into account to study magnetic island appearance and MHD activity related to rational surfaces in TJ-II.

\section{ACKNOWLEDGMENTS}

This work was supported in part by: the Spanish project MICINN ENE 2009-12213-C03-03/FTN and the Association IPP-EURATOM (the content of this publication is the sole responsibility of its authors and does not necessarily represent the views of the European Commission or its services).

One of the authors (VT) wanted to dedicate this work to Dr. Jose Guasp in his 73rd birthday.

\section{REFERENCES}

[1] C. Alejaldre et al, Fusion Technol. 13521 (1988).

[2] J. Herranz, I. Pastor, F. Castejón, E. de la Luna, I. García-Cortés, C.J. Barth, E. Ascasíbar, J. Sánchez and V. Tribaldos, Phys. Rev. Lett. 854715 (2000).

[3] J. M. Carmona, K. J. McCarthy, V. Tribaldos and R. Balbín, Fusion Sci. Technol. 54, 969 (2008).

[4] V. Tribaldos, J. A. Jiménez, J. Guasp and B. Ph. van Milligen, Plasma Phys. Control. Fusion 402113 (1998).

[5] J. Romero et al., Nucl. Fusion 43, 387 (2003).

[6] A. A. Galeev and R. Z. Sagdeev, Sov. Phys. JETP 26, 233 (1968).

[7] R. D. Hazeltine, Phys. Fluids 15, 77 (1973).

[8] C. D. Beidler, M. Yu. Isaev, S. V. Kasilov, W. Kernbichler, H. Maaßberg, S. Murakami, V. V. Nemov, D. A. Spong and V. Tribaldos, in Proceedings of the 17th International Toki Conference and 16th International Stellarator/Heliotron Workshop, Toki, 2007, edited by T. Morisaki.

[9] C. D. Beidler, K. Allmaier, M. Yu. Isaev, S. V. Kasilov, W. Kernbichler, G. O. Leitold, H. Maaßberg, D. R. Mikkelsen, S. Murakami, M. Schmidt, D. A. Spong, V. Tribaldos and A. Wakasa, Nucl. Fusion 51076001 (2011).

[10] A. H. Boozer and H. J. Gardner, Phys. Fluids B 22408 (1990).

[11] H. Maaßberg, W. Lotz, and J. Nührenberg, Phys. Fluids B 5, 3728 (1993).

[12] S. P. Hirshman, K. C. Shaing, W. I. van Rij, C. O. Beasley, Jr., and E. C. Crume, Phys. Fluids 292951 (1986).

[13] W. I. van Rij and S. P. Hirshman, Phys. Fluids B 1563 (1989).

[14] V. V. Nemov, S. V. Kasilov, W. Kernbichler, and M. F. Heyn, Phys. Plasmas 64622 (1999).

[15] W. Kernbichler, S. V. Kasilov, G. O. Leitold, V. V. Nemov, and K. Allmaier, Plasma Fusion Res. 3 S1061 (2008).

[16] C. D. Beidler, W. N. G. Hitchon, and J. L. Shohet, J. Comput. Phys. 72, 220 (1987).

[17] V. Tribaldos, Phys. Plasmas 81229 (2001).

[18] A. Wakasa, S. Murakami, H. Maaßberg, C. D. Beidler, N. Nakajima, K. Watanabe, H. Yamada, M. Okamoto, S. Oikawa, and M. Itagaki, J. Plasma Fusion Res. SERIES 4408 (2001).

[19] M. Yu. Isaev, S. Brunner, W. A. Cooper, T. M. Tran,
A. Bergmann, C. D. Beidler, J. Geiger, H. Maaßberg, J. Nührenberg, and M. Schmidt, Fusion Sci. Technol. 50 440 (2006).

[20] A. Matsuyama, and K. Hanatani, Phys. Plasmas 17 032501 (2010).

[21] K. Allmaier, S. V. Kasilov, W. Kernbichler, and G. O. Leitold, Phys. Plasmas 15072512 (2008).

[22] V. Tribaldos and J. Guasp, Plasma Phys. Control. Fusion 47545 (2005).

[23] S. P. Hirshman, W. I. van Rij, and P. Merkel, Comput. Phys. Commun. 43143 (1986).

[24] J. Nührenberg and R. Zille, Theory of Fusion Plasmas (Varenna 1987) (Editrice Compositori, Bologna, 1988), p. 3.

[25] V. Tribaldos, H. Maaßberg, J.A. Jiménez and A. Varias in Proc. $30^{t h}$ European Physics Society Conference, St. Pertersburg, Rusia, June 7-11 (2003).

[26] K. C. Shaing and J. D. Callen Phys. Fluids 263315 (1983).

[27] H. Maaßberg and C. D. Beidler and Y. Turkin, Phys. Plasmas 16072504 (2009).

[28] V. Tribaldos and B.Ph. van Milligen Nuc. Fusion 36283 (1996).

[29] B. Seiwald et al. J. Comput. Phys. 2276165 (2008).

[30] J. L. Johnson et al. Comput. Phys. Commun. 771 (1993).

[31] D. Rapisarda, B. Zurro, V. Tribaldos, A. Baciero and TJII team, Plasma Phys. Control. Fusion 49309 (2007).

[32] F. Castejón, V. Tribaldos, I. García-Cortés, E. de la Luna, J. Herranz, I. Pastor, T. Estrada and TJ-II Team, Nucl. Fusion 42271 (2002).

[33] Yu. Turkin, H. Maaßberg, C. D. Beidler, J. Geiger, and N. B. Marushchenko, Fusion Sci. Technol. 50387 (2006).

[34] Y. Turkin, C. D. Beidler, H. Maaßberg, S. Murakami, V. Tribaldos, and A. Wakasa, Phys. Plasmas 18022505 (2011).

[35] I. García-Cortés, E. de la Luna, F. Castejón, J.A. Jiménez, E. Ascasíbar, B. Brañas, T. Estrada, J. Herranz, A. López-Fraguas, I. Pastor, J. Qin, J. Sánchez, F.L. Tabares, D. Tafalla, V. Tribaldos, B. Zurro, TJ-II Team and S.V. Shchepetov in Nuclear Fusion 401867 (2000).

[36] T Estrada, E de la Luna, E Ascasíbar, J A Jiménez, F. Castejón, I. García-Cortes, A. López-Fraguas, J Sánchez and V Tribaldos, Plasma Phys. Control. Fusion 441615 
(2002)

[37] L. Krupnik, A. Alonso, E. Ascasíbar, T. Estrada, C. Hidalgo, B.Ph. van Milligen, M.A. Ochando, M.A. Pedrosa, J.L. de Pablos, V. Tribaldos, A. Chmyga, N. Dreval, G. Deshko, S. Khrebtov, A. Komarov, A. Kozachok, V. Tereshin, L. Eliseev, A. Melnikov and C. Silva, Czechoslovak Jour. Phys. 55317 (2005).

[38] F.L. Tabares, B. Brañas, I. García-Cortés, D. Tafalla, T. Estrada and V. Tribaldos Plasma Phys. Control. Fusion 431023 (2001).

[39] J. Guasp private communication.

[40] E. de la Cal, J. Guasp, A. Salas, D. Reiter, P. Borner,
J.A. Alonso, R. Balbín, D. Carralero, C. Hidalgo, J.L. de Pablos, F.L. Tabares, D. Tafalla and the TJ-II Team, Nuc. Fusion 8095005 (2008).

[41] H. Yamada, J.H. Harris, A. Dinklage, E. Ascasibar, F. Sano, S. Okamura, J. Talmadge, U. Stroth, A. Kus, S. Murakami, M. Yokoyama, C.D. Beidler, V. Tribaldos, K.Y. Watanabe and Y. Suzuki, Nucl. Fusion 451684 (2005).

[42] P. I. Strand and W. A. Houlberg, Phys. Plasmas 82782 (2001). 